\title{
Propriedades e bioatividade de um cimento endodôntico à base de aluminato de cálcio
}

\section{(Properties and bioactivity of endodontic calcium aluminate cement)}

\author{
I. R. Oliveira ${ }^{1}$, V. C. Pandolfelli ${ }^{2}$ \\ ${ }^{1}$ Universidade do Vale do Paraíba - Univap, Av. Shishima Hifumi 2911, S. José dos Campos, SP 12244-000 \\ ${ }^{2}$ Universidade Federal de S. Carlos, Rod. Washington Luiz, km 235, C.P. 676, S. Carlos, SP, 13565-905 \\ ivonero@univap.br
}

\begin{abstract}
Resumo
Desde sua introdução na endodontia como um material retro-obturador e selador de defeitos da raiz dental, o agregado de trióxido mineral (MTA) tem sido considerado como um material endodôntico revolucionário. Apesar disso, este material apresenta algumas propriedades limitantes, necessitando alterações em sua composição bem como desenvolvimento de novos materiais. Assim, o objetivo desse trabalho foi mostrar a influência de aditivos no desenvolvimento de um cimento endodôntico à base de cimento de aluminato de cálcio (ECAC). Além disso, foram avaliadas as propriedades do ECAC em comparação com o MTA, quando em contato com solução de fluido corporal simulado (SBF). Testes de manipulação e medidas de resistência à compressão, porosidade aparente, tempo de endurecimento, $\mathrm{pH}$ e condutividade iônica, foram realizados para os materiais MTA puro e ECAC contendo aditivos. Considerando as propriedades apresentadas pelo ECAC, este material alternativo pode ser indicado para múltiplas aplicações em endodontia.
\end{abstract}

Palavras-chave: cimento de aluminato de cálcio, MTA, propriedades, bioatividade.

\begin{abstract}
The mineral trioxide aggregate (MTA), a material primarily developed as a root-end filling has been extensively investigated as an innovative product for endodontic applications. However, changes in its formulation/composition involving its mineral aggregates and the development of alternatives of materials have been proposed in an attempt to overcome its negative physical-chemical characteristics. In this work, the influence of additives addition on the development of a novel endodontic cement based on calcium aluminate, has been evaluated. In addition, the properties of endodontic calcium aluminate cement (ECAC) were compared with the gold standard mineral-trioxide-aggregate in contact with simulated body fluid (SBF). Manipulation tests and measurements of compressive strength, apparent porosity, setting time, $\mathrm{pH}$ and ionic conductivity were carried out on plain MTA and calcium aluminate cement with and without various additives: a polymeric dispersant, $\mathrm{CaCl}_{2}$ as plasticizer, $\mathrm{ZnO}$ as radiopacifier and accelerator additives. Based on the ECAC properties and likely bioactivity in contact with SBF solution, this alternative material can be indicated as a potential compound for multipurpose use in endodontics.
\end{abstract}

Keywords: calcium aluminate cement, MTA, properties, bioactivity.

\section{INTRODUÇÃO}

O agregado de trióxido mineral (MTA) é um pó branco ou cinza de partículas hidrofílicas finas que endurecem na presença de umidade [1,2]. A composição do MTA é similar a do cimento Portland e ambos contém, principalmente, silicatos tri $\left(3 \mathrm{CaO} . \mathrm{SiO}_{2}\right)$ e dicálcico $\left(2 \mathrm{CaO} \mathrm{SiO}_{2}\right)$ [3]. O processo de endurecimento do MTA e cimentos Portland é descrito como uma reação de hidratação de seus silicatos sendo o $\mathrm{Ca}_{2} \mathrm{SiO}_{4}$ o responsável pelo desenvolvimento da resistência mecânica $[3,4]$. Neste processo, ocorre inicialmente a dissolução de íons a partir do material anidro que induz a formação do hidrato de silicato de cálcio (C-S-H) e hidróxido de cálcio $(\mathrm{CH})$ [4], resultando na cristalização dos hidratos com uma estrutura emaranhada que confere resistência ao material [5]. Vários estudos em laboratório e in vivo envolvendo interações entre células e tecidos com o MTA tem demostrado sua biocompatibilidade [6-8]. Entretanto, mudanças em sua composição/formulação envolvendo seus agregados minerais e o desenvolvimento de novas classes de materiais tem sido propostas visando superar algumas de suas características físico-químicas adversas $[9,10]$ que incluem: consistência arenosa (dificulta o manuseamento do material e aplicação) [11], dispersão pobre, alta porosidade, longo tempo de pega [12], escurecimento do dente e gengiva $[13,14]$ e alto custo. Neste contexto, um novo cimento endodôntico a base de cimento de aluminato de cálcio (ECAC) foi desenvolvido na Universidade Federal de S. Carlos [12] visando preservar as propriedades positivas e aplicações clínicas do MTA permitindo que suas aplicações possam ser extendidas superando as desvantagens apresentadas pelo material original, por meio da adição de aditivos específicos. 
$\mathrm{O}$ cimento de aluminato de cálcio é composto principalmente pelas fases $\mathrm{CaO} \cdot \mathrm{Al}_{2} \mathrm{O}_{3}$ e dialuminato de cálcio $\left(\mathrm{CaO} .2 \mathrm{Al}_{2} \mathrm{O}_{3}\right)$ as quais são responsáveis pelo seu processo de endurecimento hidráulico [15]. A dissolução dessas fases em contato com a água promove a liberação dos íons $\mathrm{Ca}^{2+}$, $\mathrm{Al}(\mathrm{OH})_{4}^{-}$e $\mathrm{OH}^{-}$, o que é seguido pela precipitação de hidratos de aluminato de cálcio (C-A-H) e hidróxido de alumínio (AH) devido a saturação da solução [16]. Assim, inicialmente este estudo demonstrou a influência de aditivos nas propriedades deste novo cimento endodôntico a base de aluminato de cálcio (ECAC) por meio de testes de manipulação, resistência a compressão e porosidade aparente. Em seguida foram avaliadas outras propriedades relevantes do material: seu tempo de pega quando combinado com aditivo acelerador e sua bioatividade, usando MTA como material de controle. $\mathrm{O}$ tempo de endurecimento ou pega do material necessita ser adequadamente ajustado para as aplicações clínicas pela incorporação de aditivos aceleradores os quais atuam tornando o processo de precipitação de hidratos e consequente endurecimento mais rápido. Já, a bioatividade do material se refere a sua capacidade de desenvolver uma ligação estável com tecidos vivos via a deposição de hidroxiapatita (HA) [17]. Materiais que exibem esta propriedade podem ser usados para reparar doenças ou danos no tecido ósseo e podem permanecer in situ indefinidamente. Uma indicação da bioatividade pode ser obtida pela formação de uma camada de HA na superfície de um substrato em contato com solução de fluido corporal simulado (SBF) [18].

\section{MATERIAIS E MÉTODOS}

Os cimentos hidráulicos selecionados foram cimento de aluminato de cálcio (Secar 71, Kerneos, França) e MTA-branco (Angelus, Brasil), cujas análises químicas são apresentadas na Tabela I.

Tabela I - Composição química do MTA e CAC por espectroscopia de emissão atômica com fonte de plasma acoplado indutivamente.

[Table I - Chemical compositions of MTA and calcium aluminate cement by inductively coupled plasma atomic emission spectroscopy*.]

\begin{tabular}{ccc}
\hline Fases $(\%-p)$ & MTA & CAC \\
\hline $\mathrm{Al}_{2} \mathrm{O}_{3}$ & 4,40 & 68,5 \\
$\mathrm{SiO}_{2}$ & 17,40 & 0,35 \\
$\mathrm{CaO}$ & 62,40 & 29,5 \\
$\mathrm{Na} 2$ & 0,28 & 0,47 \\
$\mathrm{SrO}$ & 0,26 & 0,02 \\
$\mathrm{MgO}$ & 0,40 & 0,07 \\
$\mathrm{Fe}_{2} \mathrm{O}_{3}$ & 0,27 & 0,07 \\
$\mathrm{Bi}_{2} \mathrm{O}_{3}$ & 12,60 & - \\
\hline
\end{tabular}

*(ICP-AES, Perkin Elmer 5300 DV, Alcoa Alumínio Brasil); obs: o $\mathrm{Bi}_{2} \mathrm{O}_{3}$ na composição do MTA atua como agente opacificante para os raios $X$.
Os aditivos usados foram: (a) um dispersante polimérico da família poliglicol (Bayer, 0,6\%-p), (b) um aditivo para induzir plasticidade ao cimento $\left(\mathrm{CaCl}_{2}\right.$, Labsynth, $2,8 \%$-p) e (c) um aditivo para fornecer radiopacidade ao cimento (ZnO, J.T. Baker, 25\%-p). Os teores de aditivos (\%-p) são em relação a massa de cimento.

Para os testes de manipulação, o cimento de aluminato de cálcio e o MTA foram colocados isoladamente sobre uma placa de vidro. Água foi adicionada a esses pós até obtenção de uma pasta homogênea. Assim, a melhor razão água/pó foi empiricamente determinada (gramas de água por gramas de cimento) a qual resultou numa consistência ideal para uso clínico. Estes testes também foram realizados na presença dos aditivos (0,6\%-p de dispersante e 2,8\%-p de plastificante) a fim de verificar a influência dos mesmos na consistência da pasta bem como no consumo de água.

Para os testes de resistência mecânica e porosidade aparente, suspensões aquosas de CAC foram preparadas na ausência ou na presença de diferentes aditivos (dispersante, plastificante e radiopaco) e colocadas em moldes cilíndricos ( $20 \mathrm{~mm}$ de diâmetro x $25 \mathrm{~mm}$ de altura). Suspensões de MTA foram preparadas apenas na ausência de aditivos. Uma razão água/cimento de 0,32 foi usada a fim de manter constante o teor de água.

As amostras foram curadas a $50{ }^{\circ} \mathrm{C}$ em uma câmara climática (Vötsch modelo 20-20) em ambiente saturado ( $\sim 100 \%$ de umidade) durante $1,7,15$, e 30 dias. Amostras úmidas (imediatamente após a cura) foram submetidas a testes de resistência a compressão em função do tempo de cura, enquanto outras foram secas a $110{ }^{\circ} \mathrm{C}$ durante $72 \mathrm{~h} \mathrm{e}$ foram usadas para medidas de porosidade aparente

Os ensaios de resistência mecânica por compressão diametral (ASTM C 496-90) foram realizados utilizandose uma máquina de ensaios universal MTS 810 e uma taxa de aplicação de carga de $11 \mathrm{~N} / \mathrm{s}$. A tensão de ruptura foi calculada a partir da equação abaixo:

$$
\sigma_{\mathrm{R}}=2 \cdot\left(\frac{\mathrm{P}_{\max }}{\pi \cdot \mathrm{L} \cdot \mathrm{D} .}\right)
$$

onde $\sigma_{R}$ é a tensão de ruptura (MPA), $\mathrm{P}_{\text {max }}$ é a força de ruptura (N), L é a altura (m) e D o diâmetro da amostra (m).

A porosidade aparente (P.A.) das amostras foi obtida pelo método de imersão de Archimedes, utilizando querosene como líquido de imersão (norma ASTM C830). Neste método as amostras são inicialmente pesadas a seco $\left(\mathrm{W}_{\mathrm{s}}\right)$. Após uma hora de imersão no líquido sob vácuo, as amostras são pesadas novamente quando imersas no líquido $\left(\mathrm{W}_{\mathrm{i}}\right) \mathrm{e}$ úmidas $\left(\mathrm{W}_{\mathrm{u}}\right)$. Assim, a porosidade aparente das amostras é calculada por meio da massa de líquido retida em seus poros abertos.

$$
\text { P.A. }=\left(\frac{(\mathrm{Wu}-\mathrm{Ws})}{(\mathrm{Wu}-\mathrm{Wi})}\right) \times 100
$$

O cimento endodôntico a base de aluminato de cálcio 
Tabela II - Quantidade de reagentes para preparação de solução de fluido corporal simulado (1L). [Table II - Reagent contents for the simulated body fluid (SBF) solution (1L) preparation.]

\begin{tabular}{cccc}
\hline Ordem & & Reagente & Quantidade \\
\hline$\# 0$ & Água & Deionizada & $750 \mathrm{~mL}$ \\
$\# 1$ & $\mathrm{NaCl}$ & PA, Quemis, Brasil & $7,996 \mathrm{~g}$ \\
$\# 2$ & $\mathrm{NaHCO}_{3}$ & PA, Quemis, Brasil & $0,50 \mathrm{~g}$ \\
$\# 3$ & $\mathrm{KCl}$ & PA, Quemis, Brasil & $0,224 \mathrm{~g}$ \\
$\# 4$ & $\mathrm{~K}_{2} \mathrm{HPO}_{4}$ & PA, Quemis, Brasil & $0,174 \mathrm{~g}$ \\
$\# 5$ & $\mathrm{MgCl}_{2} \cdot 6 \mathrm{H}_{2} \mathrm{O}$ & PA, Synth, Brasil & $0,305 \mathrm{~g}$ \\
$\# 6$ & ${\mathrm{HCl} 1 \mathrm{~mol} / \mathrm{L}}$ & $83.51 \mathrm{~mL}$ of 37\% HCl é diluído a & $40 \mathrm{~mL}$ \\
$\# 7$ & $\mathrm{CaCl}_{2} \cdot 2 \mathrm{H}_{2} \mathrm{O}$ & 1000 mL com balão volumétrico. & $0,368 \mathrm{~g}$ \\
$\# 8$ & $\mathrm{Na}_{2} \mathrm{SO}_{4}$ & PA, Synth, Brasil & $0,071 \mathrm{~g}$ \\
$\# 9$ & $\left(\mathrm{CH}_{2} \mathrm{OH}\right)_{3} \mathrm{CNH}$ & PA, Quemis, Brasil & $6,057 \mathrm{~g}$ \\
$\# 10$ & $\mathrm{HCl} 1 \mathrm{~mol} / \mathrm{L}$ & PA, Synth, Brasil & \\
\hline
\end{tabular}

(ECAC) também foi adequadamente preparado por meio da adição dos aditivos (dispersante, plastificante e radiopacificador) ao CAC seguida da homogeneização em moinho de bolas durante $1 \mathrm{~h}$. Suspensões aquosas de ECAC (75\%-p de sólidos) foram preparadas na presença de carbonato de lítio $\left(\mathrm{Li}_{2} \mathrm{CO}_{3}\right.$, Synth, $0,2 \%$-p), enquanto as suspensões de MTA foram preparadas apenas na ausência de aditivos (65\%-p de sólidos). Após mistura por 1 min usando agitador mecânico, as suspensões foram colocadas em um porta amostra $(150 \mathrm{~mL})$ para medidas do tempo de pega inicial e final usando um equipamento de registro automático Vicat (Vicamatic L27) a temperatura de $20{ }^{\circ} \mathrm{C}$. A distância de penetração da agulha na amostra foi registrada a cada 2 e 5 min para as amostras de ECAC contendo acelerador e MTA, respectivamente, até que a agulha não conseguisse mais penetrar na amostra. As suspensões também foram usadas para preparar amostras cilíndricas para medidas de pH e condutividade iônica $(10 \mathrm{~mm}$ de diâmetro x $5 \mathrm{~mm}$ de altura). A cura foi realizada a $37^{\circ} \mathrm{C}$ em uma câmara climática (Vötsch modelo 20-20) em ambiente saturado durante1 $\mathrm{h}(\mathrm{ECAC})$ e $24 \mathrm{~h}$ (MTA). Após a cura, as pastilhas foram secas a $110{ }^{\circ} \mathrm{C}$ durante $24 \mathrm{~h}$ e depositados em recipientes contendo água ou solução de fluido corporal simulado (SBF, $60 \mathrm{~mL}$ ). Sensores de $\mathrm{pH}$ e condutividade conectados à um sistema automático de aquisição de dados (ESA 9800, Matec Applied Sciences) foram inseridos no recipiente e as medidas realizadas após $0.5,2,5,7,24,72,168$ e $360 \mathrm{~h}$. A solução de fluido corporal simulado foi preparada de acordo com o procedimento descrito por Kokubo \& Takadama [19], cuja composição é apresentada na Tabela II.

\section{RESULTADOS E DISCUSSÃO}

\section{Testes de manipulação}

Uma razão água/cimento de 0,32 foi obtida por meio dos testes de manipulação para CAC sem aditivos, sendo muito próxima da obtida para o MTA puro $(0,31)$. Entretanto, a adição dos aditivos dispersante e plastificante reduziu o consumo de água resultando numa razão de 0,21 . O uso dos aditivos e consequente redução no consumo de água para a produção de uma pasta de CAC trabalhável resulta numa estrutura mais densa e consequentemente em um material mais resistente. Além disso, a redução da viscosidade bem como a plasticidade obtida podem melhorar a sua manipulação clínica, aplicação e adaptação. Assim, um importante aspecto relacionado a aplicação do CAC em endodontia é o melhoramento na reologia devido a presença do dispersante que resulta numa viscosidade de $57 \mathrm{mPa} . \mathrm{s}$ a $50 \mathrm{~s}^{-1}$ [20] superando a dificuldade de manuseamento e aplicação associadas com o MTA.

\section{Testes de resistência mecânica e porosidade aparente}

Resultados de resistência a compressão diametral e porosidade aparente, em função do tempo de cura, para amostras de CAC puro ou contendo aditivos (dispersante, plastificante e radiopacificador) comparado ao MTA puro são apresentados nas Figs. 1 e 2, respectivamente.

CAC puro (sem aditivos) apresentou resistência a compressão de 32 $\pm 3,3 \mathrm{MPa}$ após $24 \mathrm{~h}$ de cura, a qual

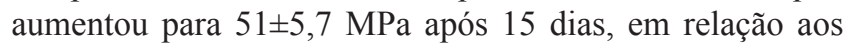
34 4 ,5 MPa medido para o MTA. Com a incorporação dos aditivos (dispersante, plastificante e radiopacificador) ao CAC, a resistência a compressão alcançou $81 \pm 3,0 \mathrm{MPa}$ (após 15 dias), enquanto para o MTA a resistência a compressão permaneceu aproximadamente $34 \pm 1,2 \mathrm{MPa}$ até mesmo após 30 dias de cura.

Após 15 dias de cura a porosidade medida para o CAC sem aditivos foi de $18 \pm 1,1 \%$, enquanto para o MTA a porosidade medida foi de $28 \pm 0,9 \%$. Quando os aditivos (dispersante, plastificante e radiopacificador) foram adicionados ao CAC a porosidade caiu para $4 \pm 0,7 \%$ após 15 dias de cura (Fig. 2). Dessa forma, além da reduzida viscosidade outras características que indicam vantagens do CAC são a sua maior resistência mecânica e baixa porosidade. A resistência 


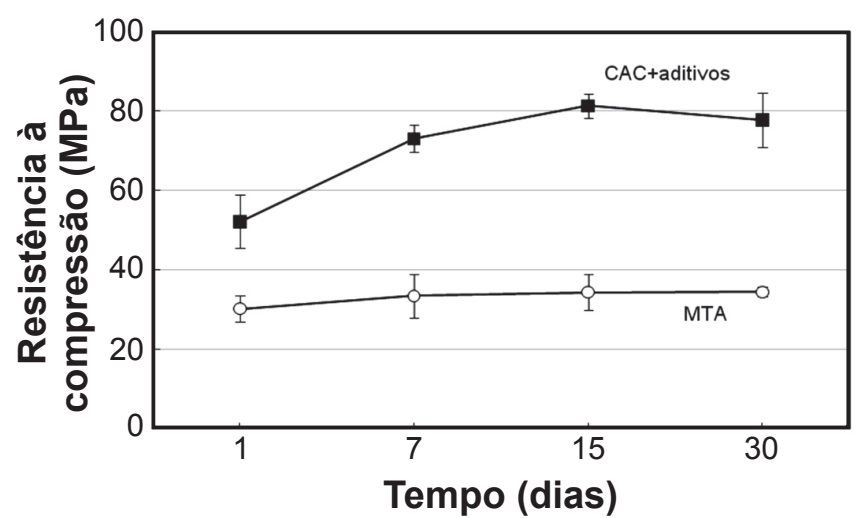

Figura 1: Resistência a compressão diametral em função do tempo de cura para amostras de MTA puro e CAC com aditivos (dipersante + plastificante + radiopacificador).

[Figure 1: Compressive strength as a function of the curing time for: samples of plain MTA and CAC with additives (dispersant+plasticizer+radiopacifier).]

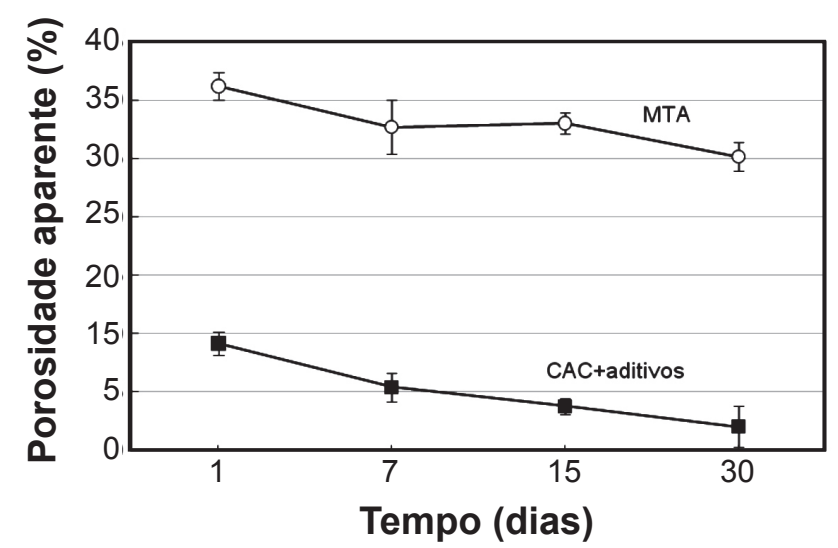

Figura 2: Porosidade aparente em função do tempo de cura para amostras de MTA puro e CAC com aditivos (dipersante + plastificante+radiopacificador).

[Figure 2: Apparent porosity as a function of the curing time for samples of plain MTA and CAC with additives (dispersant+plasticizer+radiopacifier).]

a compressão é um importante fator a ser considerado quando um material reparador é colocado em uma cavidade que irá sofrer pressão oclusal, como preenchimento de canal do dente [1] ou quando usado como base restauradora. A incorporação dos aditivos dispersante, plastificante e radiopacificador aumentou a resistência mecânica do CAC, obtendo-se quase 2,5 vezes a resistência obtida para o MTA. Além disso, a adição do radiopacificador $\mathrm{ZnO}$ apresenta uma vantagem sobre o $\mathrm{Bi}_{2} \mathrm{O}_{3}$ presente no MTA. $\mathrm{O}$ óxido de bismuto se liga ao complexo $\mathrm{CaO}-\mathrm{SiO}_{2}-\mathrm{H}_{2} \mathrm{O}$ e vai sendo retirado do material a medida que esse hidrato sofre dissolução lenta no meio [4], tornando o MTA menos radiopaco. A limitação do MTA para se obter alta resistência se deve a sua inerente porosidade a qual é associada com sua pobre dispersão e coagulação das partículas de cimento [4]. A natureza coagulada das partículas de cimento não resulta em suspensões fluidas a menos que elas sejam diluídas [4], o que aumenta a porosidade do material. A resistência a compressão do CAC foi otimizada usando um dispersante apropriado cuja ação esta relacionada a sua adsorção na superfície das partículas e consequente dispersão. Assim, um maior empacotamento de partículas pode ser obtido, além da menor razão água/cimento necessária para a produção de suspensões fluidas, o que resulta em redução da porosidade.

\section{Testes de tempo de pega}

As medidas de tempo de endurecimento (pega) inicial e final para suspensões de MTA puro e ECAC na presença de aditivo acelerador são apresentadas na Fig. 3. ECAC apresentou tempo de pega final na presença de $\mathrm{Li}_{2} \mathrm{CO}_{3}$ de 35 min, enquanto o MTA não endureceu até 180 min de ensaio.

$\mathrm{O}$ desenvolvimento de uma nova composição de CAC tem superado algumas propriedades negativas do MTA. Como visto, a adição de aditivos resultou em melhor fluidez, melhores propriedades de manuseamento, maior resistência mecânica e reduzida porosidade. Entretanto, para a obtenção de um tempo de pega adequado, a adição de aditivo acelerador é necessária. $\mathrm{O}$ cimento ECAC na presença de acelerador apresentou tempo de pega final bem menor comparado ao MTA a temperatura de $20^{\circ} \mathrm{C}$. Além disso, cabe ressaltar que o aumento da temperatura promove redução no tempo de pega do CAC [21] o que indica que esta propriedade pode ser ainda inferior em condições biológicas $\left(37^{\circ} \mathrm{C}\right)$ e assim apropriadamente ajustado para as necessidades clínicas, aproximadamente $20 \mathrm{~min}$. Por outro lado, o MTA não endurece até $400 \mathrm{~min}$ até mesmo quando a temperatura é mantida a $50{ }^{\circ} \mathrm{C}$ [20]. Uma significante redução no tempo de pega é observada na presença de $\mathrm{Li}_{2} \mathrm{CO}_{3}$ porque os ions $\mathrm{Li}^{+}$gerados a partir da dissolução desse sal favorecem a formação de compostos insolúveis como $\mathrm{LiAl}(\mathrm{OH})_{4}$, o que representa um consumo dos íons $\mathrm{Al}(\mathrm{OH})_{4}^{-}$presentes na solução. Dessa forma, os sais de lítio atuam na hidratação de cimento aumentando a concentração

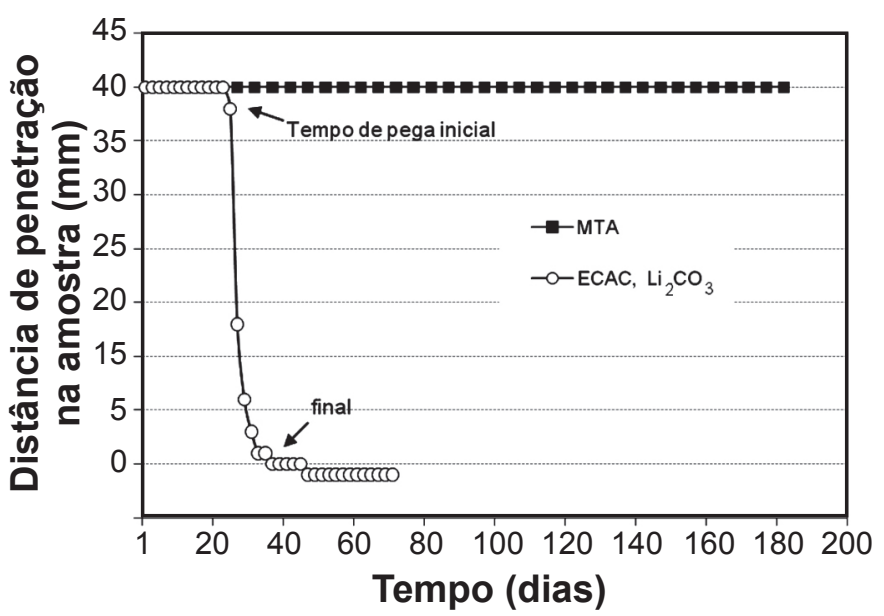

Figura 3: Avaliação do tempo de pega inicial e final para suspensões de MTA puro ou ECAC contendo $\mathrm{Li}_{2} \mathrm{CO}_{3}$. [Figure 3: Evaluation of initial and final setting times for the suspension with plain MTA or ECAC containing $\left.\mathrm{Li}_{2} \mathrm{CO}_{3}.\right]$ 
de íons cálcio no meio. Isso favorece a formação de hidratos menos solúveis (mais ricos em cálcio) e acelera o estágio de precipitação. Além disso, a precipitação desses compostos insolúveis de lítio é mais rápida do que a precipitação dos hidratos de aluminato de cálcio e apresentam estrutura cristalina necessária para servir de germes para a nucleação do hidrato de aluminato de cálcio. Isso faz com que a barreira de energia necessária à nucleação desse hidrato passe a não mais existir $[22,23]$. O uso de ECAC com reduzido tempo de pega deve adicionalmente minimizar o número de visitas ao dentista para o tratamento uma vez que os dentes podem ser restaurados em uma única sessão de tratamento. Quando usado como um material para preenchimento de canal, o rápido endurecimento deve também reduzir o risco de seu deslocamento e contaminação [4]. O endurecimento do material tão logo colocado na cavidade permite menor tempo de contato do material não endurecido com tecidos vitais [1].

\section{Testes de pH e condutividade iônica}

Uma última propriedade avaliada para os cimentos em estudo se refere à interação fisico-química da superfície desses materiais quando em contato com água e solução de fluido
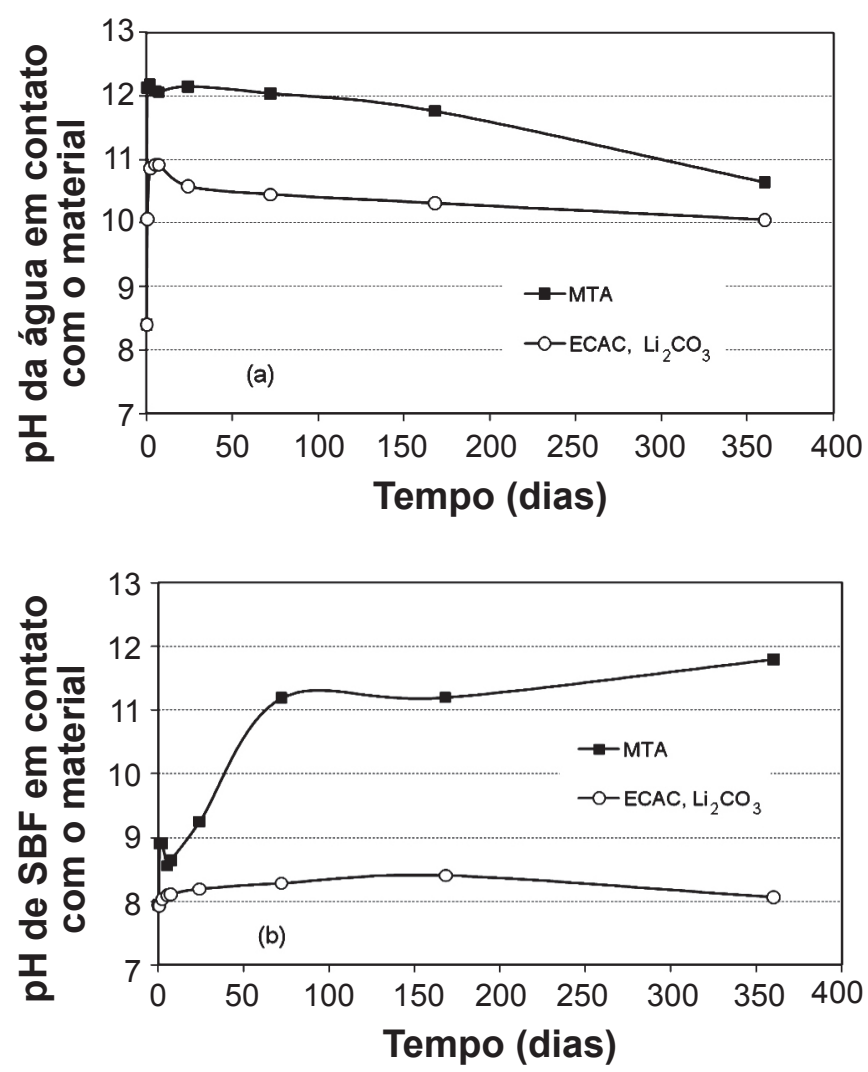

Figura 4: pH em função do tempo para (a) água e (b) solução SBF em contato com amostras endurecidas de MTA puro ou ECAC contendo $\mathrm{Li}_{2} \mathrm{CO}_{3}$.

[Figure 4: $\mathrm{pH}$ as a function of the time for (a) water and (b) SBF (simulated body fluid) in contact with set samples of plain MTA or ECAC containing $\mathrm{Li}_{2} \mathrm{CO}_{3}$.] corporal simulado (SBF). As medidas de $\mathrm{pH}$ e condutividade iônica em função do tempo para soluções em contato com amostras de MTA puro e ECAC na presença de aditivo acelerador são apresentadas nas Figs. 4 e 5, respectivamente. $\mathrm{O}$ comportamento do valor do $\mathrm{pH}$ da água após contato com os distintos materiais foi mais próximo do que quando em SBF. Em água, o pH igual a 12 foi obtido na presença de MTA tão logo a pastilha foi adicionada no líquido, enquanto $\mathrm{pH}=11$ foi medido para a água em contato com o ECAC. Por outro lado, o MTA em solução de SBF alcança valor próximo a 12 após 360 h e este é significativamente superior aquele verificado para o ECAC, $\mathrm{pH}$ 8, em todo o intervalo de tempo medido (Fig. 4).

Entretanto, as medidas de condutividade iônica mostraram comportamento contrário. Em solução de SBF os valores de condutividade iônica medidos foram mais próximos quando comparados às medidas feitas em água. MTA promoveu aumento rápido da condutividade iônica em água alcançando aproximadamente $1600 \mu \mathrm{S} / \mathrm{cm}$ após $72 \mathrm{~h}$, comparado aos $400 \mu \mathrm{S} / \mathrm{cm}$ para o ECAC, no mesmo intervalo de tempo. Após isso, a condutividade diminui resultando em um mesmo valor para ambos os materiais, após 360 h (Fig. 5).
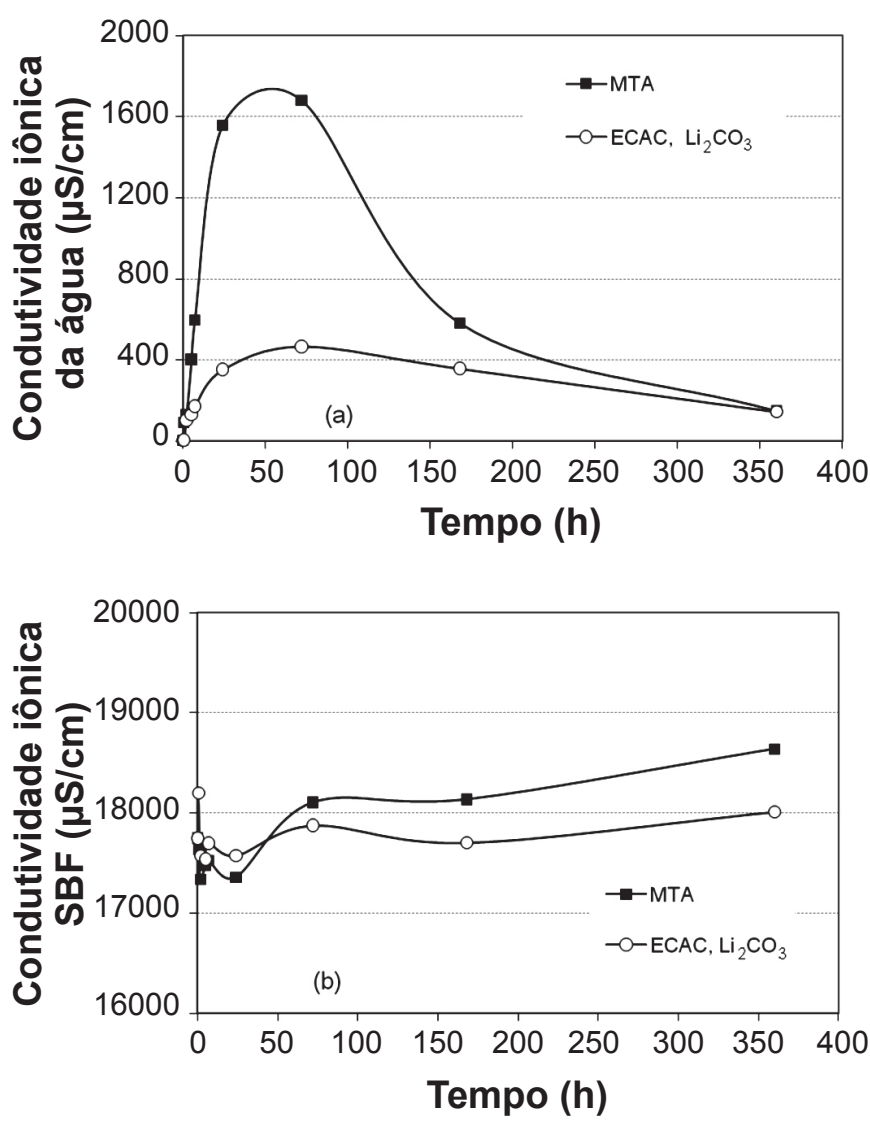

Figura 5: Condutividade iônica em função do tempo para (a) água e (b) solução SBF em contato com amostras endurecidas de MTA puro ou ECAC contendo $\mathrm{Li}_{2} \mathrm{CO}_{3}$.

[Figure 5: Ionic conductivity as a function of the time for (a) water and (b) SBF (simulated body fluid) in contact with set samples of plain MTA or ECAC containing $\mathrm{Li}_{2} \mathrm{CO}_{3}$.] 
Como visto, os produtos da hidratação do MTA são hidratos de silicato de cálcio (C-S-H) e hidróxido de cálcio (CH) [3]:

$$
\begin{aligned}
& 2\left(3 \mathrm{CaO} \cdot \mathrm{SiO}_{2}\right)+6 \mathrm{H}_{2} \mathrm{O} \rightarrow 3 \mathrm{CaO} \cdot 2 \mathrm{SiO}_{2} \cdot 3 \mathrm{H}_{2} \mathrm{O}+3 \mathrm{Ca}(\mathrm{OH})_{2}(\mathrm{C}) \\
& 2\left(2 \mathrm{CaO} \cdot \mathrm{SiO}_{2}\right)+4 \mathrm{H}_{2} \mathrm{O} \rightarrow 3 \mathrm{CaO} \cdot 2 \mathrm{SiO}_{2} \cdot 3 \mathrm{H}_{2} \mathrm{O}+\mathrm{Ca}(\mathrm{OH})_{2}(\mathrm{D})
\end{aligned}
$$

Já os cimentos de aluminato de cálcio produzem hidratos de aluminato de cálcio (C-A-H) e hidróxido de alumínio $(\mathrm{AH})$ :

$$
\begin{aligned}
& 3\left(\mathrm{CaO} . \mathrm{Al}_{2} \mathrm{O}_{3}\right)+12 \mathrm{H}_{2} \mathrm{O} \rightarrow \mathrm{Ca}_{3}\left[\mathrm{Al}(\mathrm{OH})_{4}\right]_{2}(\mathrm{OH})_{4}+ \\
& 4 \mathrm{Al}(\mathrm{OH})_{3}
\end{aligned}
$$

Depois do endurecimento, o MTA em contato com água libera uma grande quantidade de íons cálcio no meio, o que pode ser associado ao significativo aumento da condutividade iônica (Fig. 5). A fração solúvel liberada a partir do MTA é principalmente composta por hidróxido de cálcio $(\mathrm{CH})$, o qual se dissocia em íons $\mathrm{Ca}^{2+}$ e $\mathrm{OH}^{-}$, contribuindo para o aumento da condutividade e do $\mathrm{pH}$ do meio, respectivamente [24]. Já, a dissolução dos hidratos C-S-H, ocorre a uma taxa mais lenta [4]. Por outro lado, a liberação de íons $\mathrm{Ca}^{2+} \mathrm{e}$ $\mathrm{OH}^{-}$no meio pelo aluminato de cálcio endurecido pode ser atribuída apenas a dissolução dos hidratos C-A-H, uma vez que o hidróxido de alumínio é insolúvel em água. Isso poderia explicar os menores valores de $\mathrm{pH}$ e condutividade iônica da água em contato com ECAC no final do tempo de endurecimento, quando comparado ao MTA. Além disso, foi verificada uma queda acentuada na condutividade iônica da água na presença de MTA após 72 h (Fig. 5) acompanhada pelo decréscimo do pH (Fig. 4) e formação de um precipitado branco. Isso poderia ser explicado pela precipitação de carbonato de cálcio que promove o consumo dos íons $\mathrm{Ca}^{2+}$, resultantes da dissociação do $\mathrm{CH}$, resultando no decréscimo do $\mathrm{pH}$ dada a liberação de íons $\mathrm{H}^{+}$no meio, como apresentado nas reações abaixo:

$$
\begin{aligned}
& \mathrm{CO}_{2}+\mathrm{H}_{2} \mathrm{O} \leftrightarrow \mathrm{H}^{+}+\mathrm{HCO}_{3}^{-} \\
& \mathrm{Ca}^{2+}+\mathrm{HCO}_{3}^{-} \leftrightarrow \mathrm{CaCO}_{3}+\mathrm{H}^{+}
\end{aligned}
$$

Em relação ao comportamento do MTA em contato com solução SBF, a literatura tem mostrado que em solução de sais de fosfato, os íons cálcio resultantes principalmente da dissociação do hidróxido de cálcio, reagem com os íons fósforo da solução produzindo cristais de hidroxiapatita (HA) sobre a superfície das partículas de MTA. A produção de hidróxido de cálcio, liberação de íons cálcio, aumento do $\mathrm{pH}$ e por fim, a formação de HA podem ser responsáveis pela biocompatibilidade do MTA e sua capacidade seladora [24, 25]. A bioatividade do MTA tem também sido recentemente atribuída a sua abilidade de produzir HA na presença de fluidos contendo fosfatos [26]. Os íons $\mathrm{Ca}^{2+}$ liberados do MTA são pouco solúveis em fluidos biológicos resultando na precipitação de HA [25].

$$
10 \mathrm{Ca}^{2+}+6\left(\mathrm{PO}_{4}\right)^{3-}+2(\mathrm{OH})^{1-} \rightarrow \mathrm{Ca}_{10}\left(\mathrm{PO}_{4}\right)_{6}(\mathrm{OH})_{2}
$$

Esta reação é bem conhecida no processo de calcificação biológico e é favorecida em $\mathrm{pH} \mathrm{7,o} \mathrm{pH} \mathrm{do} \mathrm{fluido} \mathrm{tecidual}$ sintético. Isso pode ocorrer in vitro e in vivo com muitos materiais contendo cálcio em contato com ambiente biológico. Uma reação muito similar deve ser também responsável pela formação da camada de HA aderente na superfície do MTA [25]. Precipitação de sais de fosfato de cálcio pode também ocorrer se o cimento endurecido está em contato com fluidos corporais contendo fosfatos. Quando o MTA endurecido é hidratado, C-S-H e principalmente $\mathrm{CH}$ suprem cálcio e ions $\mathrm{OH}^{-}$para a precipitação de fosfato de cálcio. O fluido corporal simulado (SBF) usado neste trabalho apresenta concentrações de íons inorgânicos similares aquela do fluido extracelular humano (plasma humano), a fim de reproduzir a formação de apatitas sobre materiais bioativos in vitro. SBF é uma solução metaestável contendo íons cálcio e fosfato já supersaturados com relação a apatita [19]. A presença de certos grupos funcionais, tal como $\mathrm{Si}-\mathrm{OH}$, na superfície do material, induz a nucleação de cristais de apatita os quais continuam a crescer espontaneamente [18]. A camada superficial de HA resultante é similar em estrutura aos componentes minerais do osso e representa um ponto para a aderência e proliferação de novas células formadoras de osso [18]. Um material com uma camada de apatita em sua superfície que está em proximidade com tecidos calcificados como dentes e osso forma uma ligação química e integração biológica com eles [25]. Assim, esse material poderia ser usado para restaurar os dentes mais próximos ao seu estado original, comparado a materiais restauradores como a amálgama. A precipitação de HA na superfície de um material exposto a solução SBF in vitro fornece uma indicação se o material é bioativo [28]. Este fluido pode ser usado não apenas para avaliar a bioatividade de materiais artificiais in vitro, mas também recobrir com apatita vários materiais sobre condições biomiméticas [19].

Também tem sido verificado por análise de difração de raios X que a pasta de cimento Portland branco após a imersão em SBF apresenta formação de HA e aumento na proporção de calcita $\left(\mathrm{CaCO}_{3}\right)$, quando comparado a hidratação em água. Além disso, o aumento do $\mathrm{pH}$ é atenuado pelo consumo de íons $\mathrm{OH}^{-}$da dissolução do hidróxido de cálcio para a formação da estrutura da HA [18]. Dessa forma, o consumo dos íons $\mathrm{Ca}^{2+} \mathrm{e} \mathrm{OH}^{-}$provenientes do MTA para a formação de HA pode explicar o fato da condutividade não ter aumentado como observado na água, além do menor valor de $\mathrm{pH}$ alcançado inicialmente em $\operatorname{SBF}(9,0)$ comparado a água (12). Entretanto, com o passar do tempo, o pH em SBF volta a aumentar alcançando valor próximo de 12 enquanto a condutividade apresenta um aumento mais lento. Considerando que o consumo de íons $\mathrm{OH}^{-}$é menor para a formação de HA, $\left[\mathrm{Ca}_{10}\left(\mathrm{PO}_{4}\right)_{6}(\mathrm{OH})_{2}\right]$, isso poderia explicar o aumento do $\mathrm{pH}$ na solução SBF com o tempo, a medida que mais íons são liberados pela dissociação do $\mathrm{CH}$ e não são consumidos totalmente na formação de HA. No caso do $\mathrm{Ca}^{2+}$ seu consumo é bem superior ao $\mathrm{OH}^{-}$, contribuindo pouco para o aumento da condutividade com o passar do tempo. No caso do ECAC, a literatura mostra que se íons fosfato, $\mathrm{Ca}^{2+}, \mathrm{Al}(\mathrm{OH})_{4}^{-}$e $\mathrm{OH}^{-}$ estiverem presentes durante o estágio de precipitação, HA 
pode também ser formada [27]. De fato, no caso do ECAC também foi observado que o $\mathrm{pH}$ alcança menor valor inicial em $\operatorname{SBF}(8,0)$ comparado em água (11) indicando que a formação de HA também está ocorrendo. Neste caso, como a formação dos íons é consideravelmente menor comparado ao MTA, não foi observado aumento posterior permanecendo em $\mathrm{pH}=8$. No caso do MTA o alto valor de $\mathrm{pH}$ alcançado em SBF é responsável pela sua atividade antibactericida uma vez que bactérias não sobrevivem em $\mathrm{pH}$ altamente alcalino (superior a 9,5). Já, considerando o pH do ECAC em SBF $(8,0)$ este material apresenta um poder antibactericida menor. Por outro lado, isso abre novas perspectivas de aplicação desse material. Na ortopedia, além de não produzir calor durante a pega, outro requisito ideal do cimento para reparação óssea é a de desenvolver um $\mathrm{pH}$ neutro $(6,5-8,5)$ durante e depois da pega para evitar efeitos citotóxicos. Dessa forma, dada a provável capacidade do ECAC também induzir a formação de HA, ainda que menos favorável do que o MTA, este material pode ser considerado bioativo.

\section{CONCLUSÕES}

O cimento endodôntico a base de aluminato de cálcio (ECAC) possui propriedades intrínsecas tais como: (i) curto tempo de pega quando apropriadamente combinado com $\mathrm{Li}_{2} \mathrm{CO}_{3}$ e (ii) capacidade de liberar íons $\mathrm{Ca}^{2+}$ e $\mathrm{OH}^{-}$ alcalinizando o meio. $\mathrm{O}$ desenvolvimento da composição do ECAC por meio da adição de aditivos específicos também superou algumas características negativas do MTA, resultando em melhor fluidez, melhores condições de manuseamento, maior resistência mecânica e reduzida porosidade, quando comparado ao MTA. Além disso, com base em medidas de $\mathrm{pH}$ e condutividade iônica, o ECAC apresenta-se como um material bioativo em um ambiente biológico simulado. Quando o material é colocado em contato com SBF, íons $\mathrm{Ca}^{2+}$ e $\mathrm{OH}^{-}$são liberados acentuando a supersaturação da solução o que pode induzir a precipitação de hidroxiapatita sobre a superfície das partículas. A exemplo do que ocorre no MTA, a deposição de HA também pode ser atribuída a pre-existência de sítios de nucleação (Al-OH) presentes nos hidratos de aluminato de cálcio. Por outro lado, a superior concentração de ions $\mathrm{Ca}^{2+}$, gerados pela dissolução do hidróxido de cálcio deve tornar mais favorável a formação de HA quando MTA é colocado em contato com solução de SBF.

\section{AGRADECIMENTOS}

À FAPESP e ao CNPq pelo suporte financeiro.

\section{REFERÊNCIAS}

[1] M. Torabinejad, C. U. Hong, F. McDonald, T. R. Pitt Ford, J. Endodontics 21 (1995) 349-353.

[2] A. D. Santos, J. C. Moraes, E. B. Araújo, K. Yukimitu, W. V. Valério F., Int. Endodontic J. 38 (2005) 443-447.

[3] T. Dammaschke, H. U. V. Gerth, H. Zuchner, E. Schafer,
Dental Mater. 21 (2005) 731-738.

[4] J. Camilleri, Int. Endodontic J. 41 (2008) 843-849.

[5] H. W. Roberts, J. M. Toth, D. W. Berzins, D. G. Charlton, Dental Mater. 24 (2008) 149-164.

[6] E. T. Koh, M. Torabinejad, T. R. Pitt Ford, K. Brady, F. McDonald, J. Biomedical Mater. Res. 37 (1997) 432-439.

[7] E. T. Koh, F. McDonald, T. R. Pitt Ford, M. Torabinejad, J. Endodontics 24 (1998) 543-547.

[8] P. J. Mitchell, T. R. Pitt Ford, M. Torabinejad, F. McDonald, Biomater. 20 (1999) 167-173.

[10] E. A. Bortoluzzi, N. J. Broon, C. M. Bramante, A. Consolaro, R. B. Garcia, I. G. Moraes, N. Bernardineli, J. Endodontics 34 (2008) 172-175.

[11] B. S. Ber, J. F. Hatton, G. P. Stewart, J. Endodontics 33 (2007) 1231-1234.

[12] V. C. Pandolfelli, I. R. Oliveira, H. L. Rossetto, M. Jacobovitz, "Composição à base de cimento aluminoso para aplicações em endodontia e produto cimentício obtido", Registro de patente INPI 0704502-6, Universidade Federal de S. Carlos, SP (2007).

[13] E. A. Bortoluzzi, G. S. Araújo, J. M. Guerreiro Tanomaru, M. Tanomaru-Filho, J. Endodontics 33 (2007) 325-327.

[14] M. Jacobovitz, R. K. P. de Lima, Int. Endodontic J. 41 (2008) 905-912.

[15] K. M. Parker, J. H. Sharp, Brit. Ceram. Trans. J. 81 (1982) 35-42.

[16] C. Alt, L. Wong, C. Parr, Refractories Appl. News 8 (2003) 15-18.

[17] B. Czarnecka, N. J. Coleman, H. Shaw, J. W. Nicholson, Dental Medical Problems 45 (2008) 5-11.

[18] N. J. Coleman, J. W. Nicholson, K. Awosanya, Cement Concrete Res. 37 (2007) 1518-1523.

[19] T. Kokubo, H. Takadama, Biomater. 27 (2006) 29072915.

[20] I. R. Oliveira, V. C. Pandolfelli, Ceram. Int. 35 (2009) 1453-1460.

[21] I. R. Oliveira, F. S. Ortega, L. R. M. Bittencourt, V. C. Pandolfelli, J. Tech. Assoc. Refract. (Japan) 28 (2008) 172179.

[22] B. R. Currell, R. Grzeskowlak, H. G. Midgley, J. R. Parsonage, Cement and Concrete Res. 7 (1987) 420-432.

[23] I. R. Oliveira, V. C. Pandolfelli, Refractories Appl. News 14 (2009) 11-18.

[24] M. Parirokh, S. Asgary, M. J. Eghbal, J. Ghoddusi, F. Brink, S. Askarifar, M. Torabinejad, M. Raoof, Int. Endodontic J. 2 (2007) 81-86.

[25] N. K. Sarkar, R. Caicedo, P. Ritwik, R. Moiseyeva, I. Kawashima, J. Endodontics 31 (2005) 97-100.

[26] F. R. Tay, D. H. Pashley, F. A. Rueggeberg, R. J. Loushine, R. N. Weller, J. Endodontics 33 (2007) 1347-1351.

[27] H. Engqvist, J. E. Schultz-Walz, J. Loof, G. A. Botton, D. Mayer, M. W. Phaneuf, N. O. Ahnfelt, L. Hermansson, Biomater. 25 (2004) 2781-2787.

[28] X. Wang, H. Sun, J. Chang, Dental Mater. 24 (2008) 7482.

(Rec. 05/08/2010, Ac. 30/12/2010) 